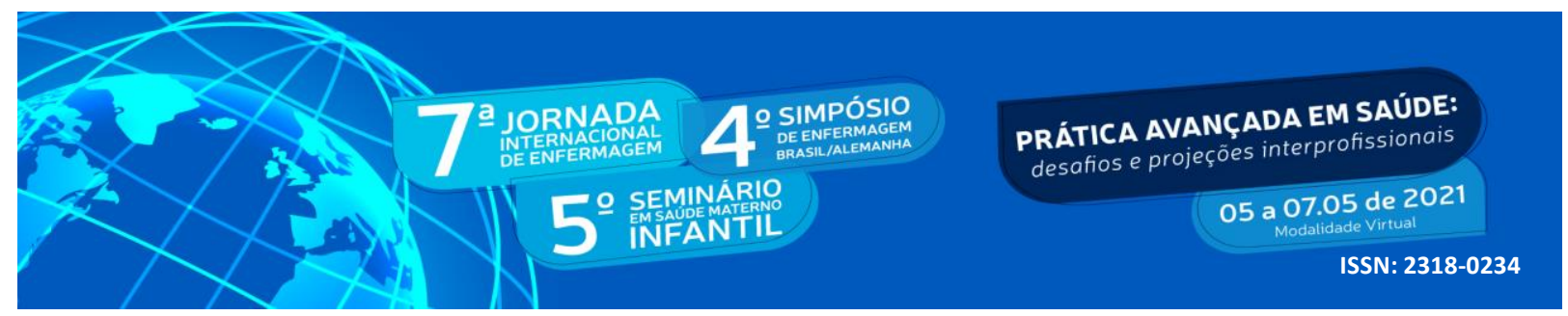

DOI: http://doi.org/10.48195/jie2021-098

\title{
AÇÕES DO ENFERMEIRO NO CUIDADO À GESTANTE NA ATENÇÃO PRIMÁRIA À SAÚDE: UMA REVISÃo INTEGRATIVA ${ }^{1}$
}

\author{
Maria de Lourdes Alves da Cruz ${ }^{2}$; Larissa Beatriz Francisca de Souza ${ }^{3}$; Ana Luiza \\ Santos Quirino ${ }^{4}$; Sidney Bruno Lima da Silva ${ }^{5}$; Renata Marinho Fernandes ${ }^{6}$; Ana Luisa \\ Brandão de Carvalho Lira ${ }^{7}$
}

\begin{abstract}
RESUMO
Objetivou-se identificar na literatura as ações de enfermagem no cuidado à gestante na atenção primária à saúde. Trata-se de uma revisão integrativa da literatura, realizada em fevereiro de 2021, nas fontes de dados: Biblioteca Virtual em Saúde, Scopus Content Overview, Cumulative Index to Nursing and Allied Health Literature e PubMed. Foram identificados 778, selecionando-se 9 artigos para compor a amostra. Em relação aos resultados, a maioria dos artigos eram estudos nacionais $(77,8 \%$ ), o ano de 2020 contribuiu com $44,4 \%$ das publicações e o método que prevaleceu foram estudos descritivos com abordagem qualitativa $(77,8 \%)$. Evidenciou-se que as principais ações realizadas pelo enfermeiro foram: prescrição de medicamentos, orientações nutricionais e orientações sobre cuidados com o bebê, solicitação de exames, encaminhamento para atenção secundária e educação em saúde. Concluiu-se que as ações realizadas contribuem para promoção e prevenção da saúde materno-infantil.
\end{abstract}

Palavras-chave: Atenção Primária à Saúde; Cuidados de Enfermagem; Gestação.

\begin{abstract}
The objective of this study was to identify nursing actions in the care of pregnant women in primary health care in the literature. This is an integrative literature review, carried out in February 2021, on the data sources: Virtual Health Library, Scopus Content Overview, Cumulative Index to Nursing and Allied Health Literature and PubMed. 778 were identified, selecting 9 articles to compose the sample. Regarding the results, most of the articles were national studies (77.8\%), the year 2020 contributed $44.4 \%$ of the publications and the method that prevailed was descriptive studies with a qualitative approach (77.8\%). It was evidenced that the main actions performed by the nurse were: prescription of medications, nutritional guidance and guidance on baby care, request for exams, referral to secondary care and health education. It was concluded that the actions carried out contribute to the promotion and prevention of maternal and child health.
\end{abstract}

Key Words: Nursing Care; Pregnancy; Primary Health Care.

\footnotetext{
${ }^{1}$ Revisão Integrativa da Literatura.

2 Estudante do Curso de Graduação em Enfermagem.UFRN. E-mail: maria_lourdes@ hotmail.com.

3 Estudante do Curso de Graduação em Enfermagem.UFRN. E-mail: larissa.beatriz@ufrn.edu.br.

${ }^{4}$ Estudante do Curso de Graduação em Enfermagem. UFRN. E-mail: luizasantos699@ gmail.com

${ }^{5}$ Estudante do Curso de Graduação em Enfermagem.UFRN. E-mail: sidney.natal10@gmail.com

${ }^{6}$ Mestra em Enfermagem. UFRN. E-mail: remarifernandes@gmail.com.

${ }^{7}$ Orientadora. Doutora em Enfermagem. UFRN. E-mail: analuisa_brandao@yahoo.com.br.
} 


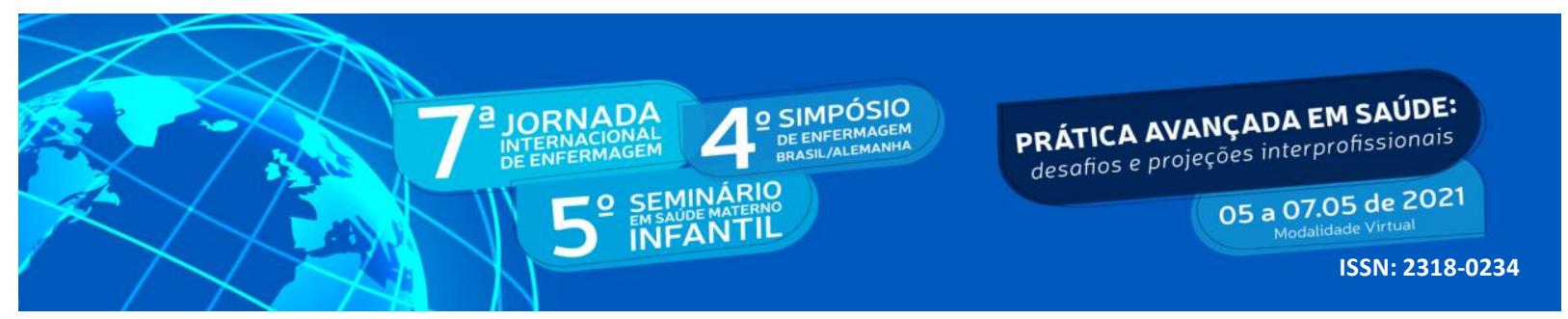

\section{INTRODUÇÃO}

O período gestacional é caracterizado por alterações fisiológicas, psicológicas e sociais na mulher, estando diretamente ligada à existência humana. Esse período tende a tornar a mulher mais vulnerável, necessitando de orientações e acompanhamento pela família e profissionais da saúde, para que este momento seja vivenciado com segurança e tranquilidade (DIAS et al., 2018).

Dessa maneira, torna-se fundamental a assistência em saúde como estratégia para a prevenção de complicações ao longo da gestação e parto. Para tanto, salienta-se que a assistência ao período gestacional deve ser realizada em três fases: gestação, parto e puerpério (BALSELLS et al., 2018).

Nessa perspectiva, insere-se a assistência pré-natal como uma estratégia para o cuidado holístico nesse período, além de ser responsável pela diminuição da morbimortalidade materna e perinatal, sendo uma das prioridades na agenda global da Organização Mundial da Saúde (OMS) (OMS, 2016; SIMÃO et al., 2019).

Em consonância, a melhoria da saúde materna e a redução das taxas de morbimortalidade materno-infantil fazem parte dos Objetivos de Desenvolvimento do Milênio (ONU, 2015). Todavia, dados da OMS evidenciam que, entre os anos de 2007 e 2014, apenas $64 \%$ das gestantes compareceram às consultas mínimas recomendadas, sugerindo reflexão quanto aos cuidados realizados (OMS, 2016).

Nesse sentido, destaca-se a Atenção Primária à Saúde (APS), pois se configura como um espaço oportuno para o cuidado integral e de qualidade às mulheres (MARQUES et al., 2018). A APS é responsável pela inserção dos usuários aos demais níveis de atenção e pelas práticas de promoção, prevenção e recuperação da saúde (IPUCHIMA; SOUZA; WEIS, 2017).

À vista disso, as gestantes são assistidas por meio do pré-natal, que é uma estratégia de acompanhamento gestacional imprescindível para prevenir e diminuir os fatores de riscos, educação e promoção da saúde, tratamento de complicações e a redução da mortalidade do binômio mãe-filho, pela equipe de saúde, dentre ela o enfermeiro (DYESS-NUGENT, 2018).

Neste seguimento, compreende-se que a assistência do enfermeiro na APS engloba o indivíduo, a família e a comunidade e vem se configurando como instrumento para mudanças 


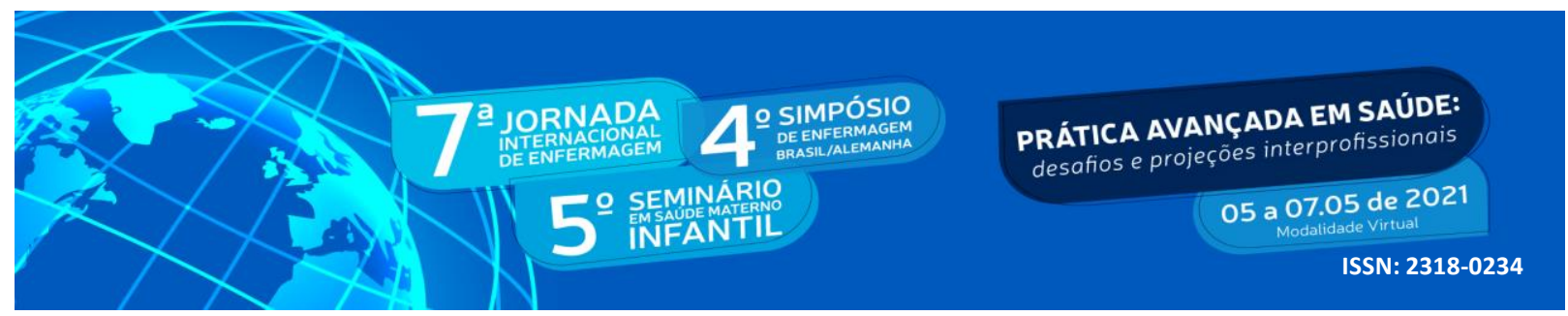

na prática assistencial e para a consolidação do Sistema Único de Saúde (SUS) (FERREIRA; PÉRICO; DIAS, 2018). Tal profissional detém papel fundamental na integração do sistema de cuidado por meio de práticas gerencial, assistencial e educacional (DYESS-NUGENT, 2018).

Para mais, observa-se que o cuidado de enfermagem abrange diversos aspectos na assistência ao paciente, sendo este o centro do cuidado, considerando-o em todos seus aspectos biopsicosocioespiritual (AGYEMAN-YEBOAH; KORSAH; OKRAH, 2017). Outrossim, por meio do desenvolvimento de um conjunto de ações direcionadas à gestante, o enfermeiro auxilia na redução de riscos e agravos à saúde materna-infantil (GOMES et al., 2019).

Diante do exposto, o presente estudo se justifica por evidenciar a importância das ações de enfermagem na atenção primária para contribuir com a promoção da saúde às gestantes e identificar os benefícios e possíveis fragilidades dessa assistência na promoção da saúde da mulher.

\section{OBJETIVO}

Identificar na literatura as ações de enfermagem no cuidado à gestante na atenção primária à saúde.

\section{METODOLOGIA}

Trata-se de uma revisão integrativa da literatura, método utilizado para analisar temáticas, de maneira organizada e sintética, viabilizando a compreensão de um determinado assunto e a identificação de possíveis lacunas do conhecimento (CARVALHO et al., 2018).

Para direcionar este estudo foi elaborado um protocolo de busca contendo as informações a seguir: título; objetivo; questão norteadora; critérios de inclusão e exclusão; estratégia para busca e seleção dos estudos; método para seleção dos dados; síntese e apresentação dos dados. Delimitou-se a seguinte questão norteadora: “Quais são as estratégias utilizadas pelo enfermeiro no cuidado à gestante na atenção primária à saúde?”.

A coleta ocorreu em fevereiro de 2021, na qual se buscou artigos nas fontes de dados Scopus Content Overview (SCOPUS), Cumulative Index to Nursing and Allied Health 


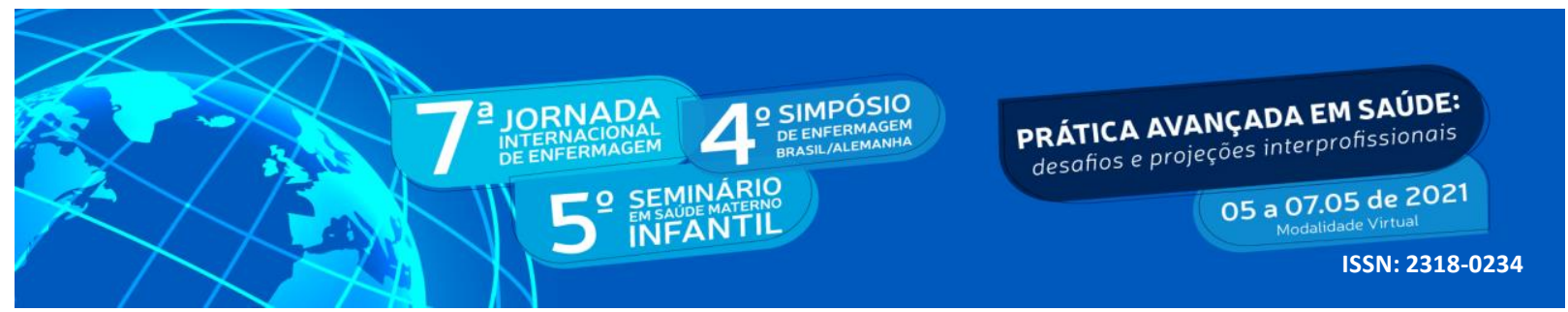

Literature (CINAHL), PubMed e Biblioteca Virtual em Saúde (BVS), acessadas por meio do proxy da Universidade Federal do Rio Grande do Norte (UFRN) no CAFe da CAPES.

Os critérios para inclusão foram: artigos completos, gratuitos e disponíveis na íntegra, que contemplassem a temática, escritos em qualquer idioma e publicados nos últimos cinco anos (2017-2021), visando obter conhecimentos atuais. Foram excluídos editoriais, resumos, relatos de experiências, dissertações, teses, revisões da literatura e artigos duplicatas nas fontes de dados selecionadas.

Realizou-se uma consulta no Medical Subject Headings (MeSH) e nos Descritores em Ciências da Saúde (DeCS), elencando-se os seguintes descritores controlados, a saber: "Cuidados de Enfermagem”, "Nursing Care”, "Gravidez”, "Pregnancy”, “Atenção Primária à Saúde" e "Primary Health Care", associados ao operador booleano AND.

A busca foi realizada simultaneamente por dois pesquisadores, em computadores distintos, utilizando como apoio a ferramenta computacional State of the Art Through Systematic Review (StArt) que permite organizar de forma metódica o desenvolvimento das revisões da literatura. Em caso de discordâncias, houve discussão entre os avaliadores até o consenso.

Inicialmente, foram encontradas 778 publicações e, após aplicação dos critérios de elegibilidade, houve redução para 187 artigos. Realizou-se leitura seletiva dos títulos e resumos desses artigos com a finalidade de refinar a amostra, selecionando-se 27 artigos para leitura na íntegra. Após análise dos estudos, por meio da leitura criteriosa dos textos, 9 artigos foram selecionados para compor este estudo. Para melhor visualização do método utilizado foi construído um fluxograma (Figura 1).

Figura 1: Fluxograma do método utilizado. 

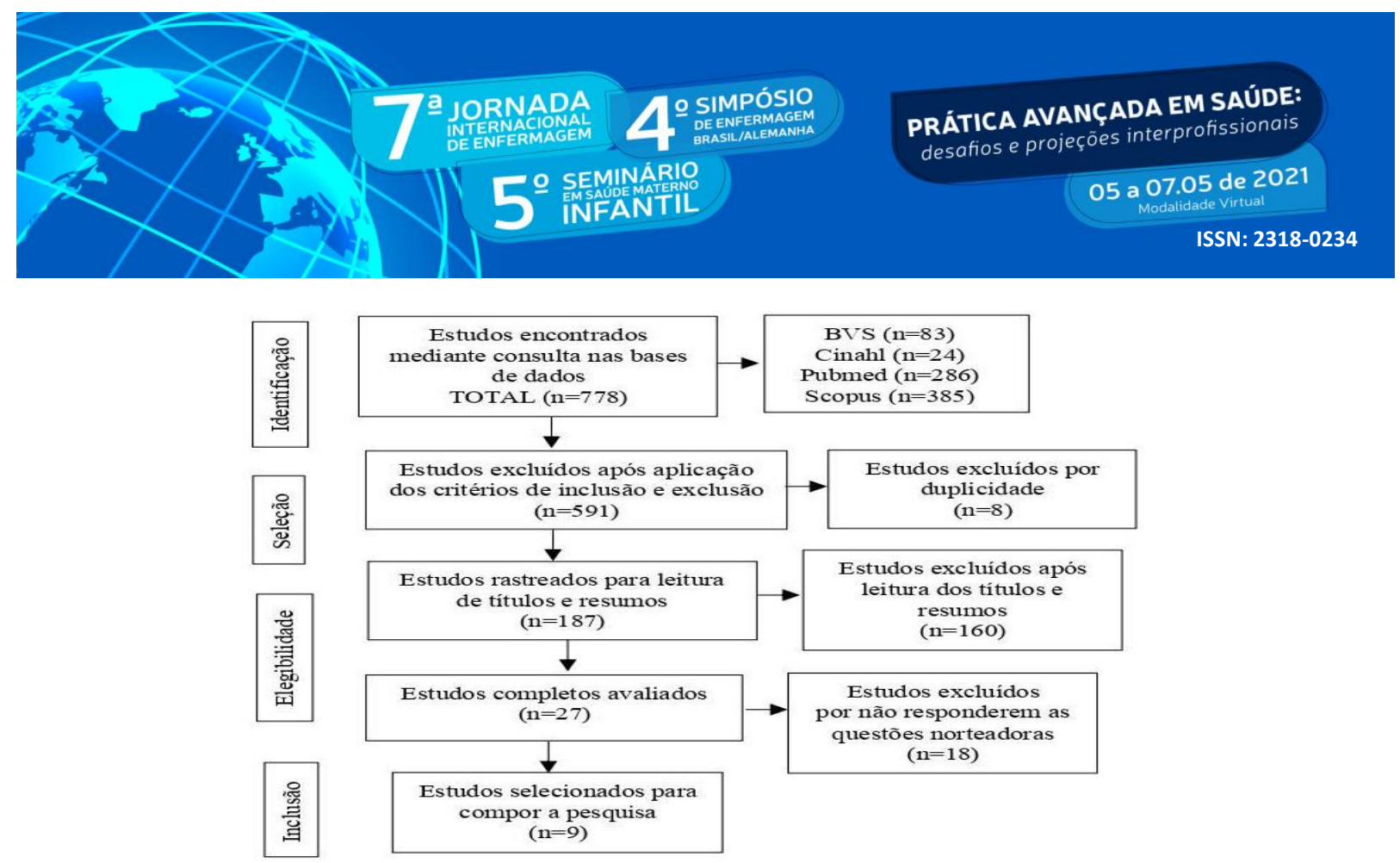

Fonte: Autores.

\section{RESULTADOS E DISCUSSÃO}

Dos estudos incluídos na revisão integrativa, 77,8\% foram pesquisas realizadas em âmbito nacional e 22,2\% eram pesquisas internacionais. O ano de 2020 teve o maior quantitativo de publicações (44,4\%), seguido de 2017 (33,3\%) e 2019 (22,2\%). Em relação ao tipo de delineamento dos estudos avaliados, $77,8 \%$ foram estudos descritivos e com abordagem qualitativa. Para sintetizar as características dos manuscritos incluídos na revisão foi construída uma tabela (Tabela 1).

Tabela 1. Síntese dos manuscritos incluídos na revisão.

\begin{tabular}{clll}
\hline \multicolumn{1}{c}{ Autores/ano } & \multicolumn{1}{c}{ Método } & \multicolumn{1}{c}{ Principais ações } \\
\hline Chaves et al., 2020 & $\begin{array}{l}\text { Estudo descritivo } \\
\text { abordagem } \\
\text { qualitativa }\end{array}$ & $\begin{array}{l}\text { de } \\
\text { enantitativa }\end{array}$ & $\begin{array}{l}\text { Acolhimento, escuta qualificada, educação } \\
\text { em saúde, orientação quanto ao uso de } \\
\text { medicamentos }\end{array}$ \\
Peters et al., 2020 & $\begin{array}{l}\text { Estudo descritivo-exploratório } \\
\text { de abordagem qualitativa }\end{array}$ & $\begin{array}{l}\text { Encaminhamento para atenção secundária e } \\
\text { assistente social, grupos educativos }\end{array}$ \\
Piro; Ahmed, 2020 & Estudo experimental & $\begin{array}{l}\text { Explicação sobre amamentação por meio de } \\
\text { cartilhas e vídeos educativos e } \\
\text { aconselhamentos }\end{array}$ \\
Piro; Ahmed, 2020 & Estudo experimental & $\begin{array}{l}\text { Estratégias educacionais acerca do impacto } \\
\text { da amamentação }\end{array}$
\end{tabular}




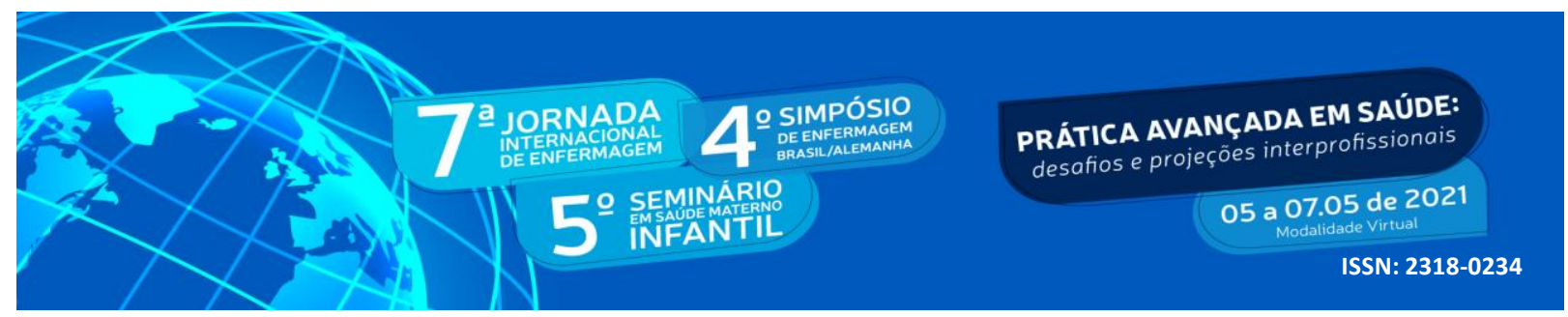

Gomes et al., 2019 Estudo qualitativo, exploratório Acolhimento, encaminhamento médico, e descritivo orientações quanto a alimentação e uso de medicamentos

Rocha et al., 2019 Estudo qualitativo, descritivo, Atividades em grupos para escuta, diálogo, exploratório

troca de experiências com as gestantes

Araújo et al., 2017 Estudo descritivo, com Orientações quanto aos medicamentos, abordagem qualitativa alimentação, repouso e posicionamento para dormir

Bortoli et al., 2017 Estudo qualitativo, de campo, Prescrição e orientação para uso de do tipo descritivo

medicamentos, solicitação de exames de rotina, encaminhamento para unidade de referência

Sousa et al., 2017 Estudo descritivo com Prescrição de exames, orientações e abordagem qualitativa encaminhamento para tratamento especializado

Fonte: Autores.

A literatura evidencia que o cuidado ofertado à gestante ao longo do pré-natal reduz a morbimortalidade materno-infantil (DYESS-NUGENT, 2018; KIM; KIM, 2020). No entanto, observa-se que a assistência pré-natal ainda é deficiente, principalmente, em nações subdesenvolvidas e que estão em desenvolvimento (GARCIA et al., 2018).

No Brasil, o comprometimento com essa causa vem ocorrendo nos últimos anos, com a instituição de políticas de atenção à saúde materno-infantil como o Programa Rede Cegonha, criado em 2011, que visa garantir às mulheres planejamento reprodutivo e atenção humanizada à gravidez, através de ações para ampliação e qualificação do acesso ao pré-natal (MAYOR et al., 2018).

Nessa conjuntura, a assistência realizada pelo enfermeiro durante o pré-natal, na APS, refere-se à gravidez classificada como baixo risco ou risco habitual, no entanto, esse profissional também tem a responsabilidade de acompanhar o pré-natal de alto risco (LIVRAMENTO et al., 2019). O pré-natal de risco habitual se refere à gestação em que a ocorrência de complicações são mínimas, já no pré-natal de alto risco as gestantes apresentam algumas afecções específicas da gravidez, necessitando de atenção específica pelos profissionais ao longo da gestação, parto e puerpério (LIMA et al., 2019)

Dessa maneira, conforme recomendações do Ministério da Saúde (MS), a assistência 


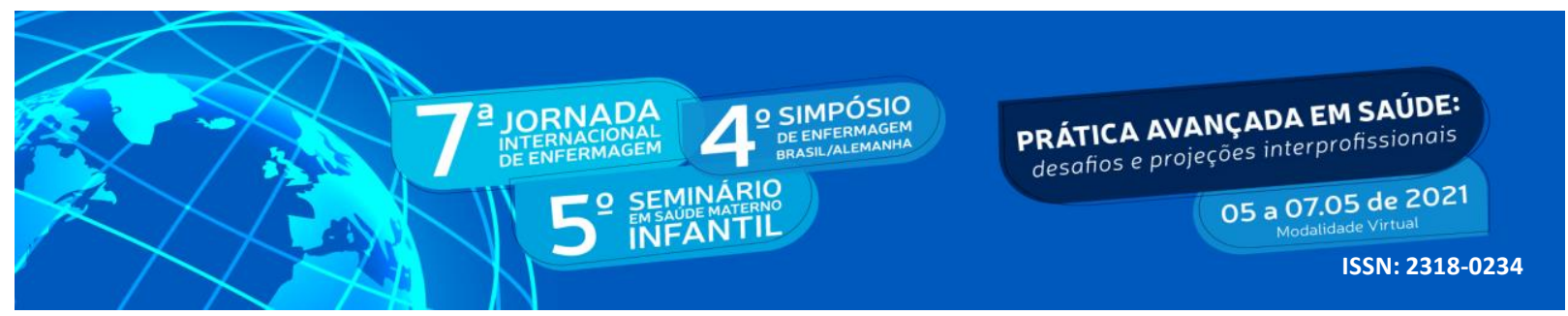

pré-natal deve ser realizada em, no mínimo, seis consultas, por meio de condutas acolhedoras, ações preventivas, atividades educativas em grupo ou individual (BRASIL, 2006). Corroborando com tais recomendações, Liu et al. (2017) afirmam que a atenção realizada pelo enfermeiro, no pré-natal, deve contemplar as práticas educativas e assistenciais para tornar o período gestacional mais saudável e seguro.

Para tanto, faz-se necessário o estabelecimento do vínculo entre o profissional, o usuário e sua família, visto que a segurança e confiança construída nessa relação infere diretamente no cuidado ofertado (BORTOLI $e$ al., 2017). Em consonância, estudos evidenciam que na percepção das gestantes o vínculo criado com o enfermeiro é elemento fundamental para a continuidade e qualidade da assistência, por compreender o indivíduo de maneira holística (CHAVES et al., 2020; GOMES et al., 2019).

Nesse seguimento, insere-se o acolhimento, que é uma ação de cuidado realizada pelo enfermeiro, durante o acompanhamento à gestante por meio da escuta qualificada, permitindoa expressar suas angústias e preocupações, bem como assegurar a resolução das queixas e promover a autonomia da mulher (CHAVES et al., 2020; BORTOLI $e$ al., 2017). Estudo afirma que na perspectiva das gestantes, o acolhimento, é um aspecto essencial para adesão das consultas, compreensão do período gravídico-puerperal e estabelecimento do vínculo profissional-usuário (BORTOLI et al., 2017).

Verificou-se como ações de enfermagem recorrentes, na APS, presentes na literatura, para avaliação bem-estar materno-fetal, exame físico na gestante, prescrição de medicamentos como ácido fólico e sulfato ferroso, solicitação de exames, verificação do caderno de vacinação da gestante, encaminhamento para o médico e para serviço de referência (BORTOLI $e$ al., 2017; CHAVES et al., 2020; GOMES et al., 2019; SOUSA et al., 2017).

As estratégias de educação em saúde identificadas na literatura abordam orientações nutricionais e os cuidados com o bebê, amamentação, repouso e posicionamento para dormir (GOMES et al., 2019; CHAVES et al., 2020; ARAÚJO et al., 2017). Nessa direção, salientase a educação em saúde como estratégia para prevenção e controle de possíveis complicações durante a gravidez, parto e puerpério, por meio da explanação das necessidades, riscos e os cuidados que as gestantes devem ter nesse período (PETERS et al., 2020).

Ainda, estudo realizado com gestantes e enfermeiros por meio de ações educativas 


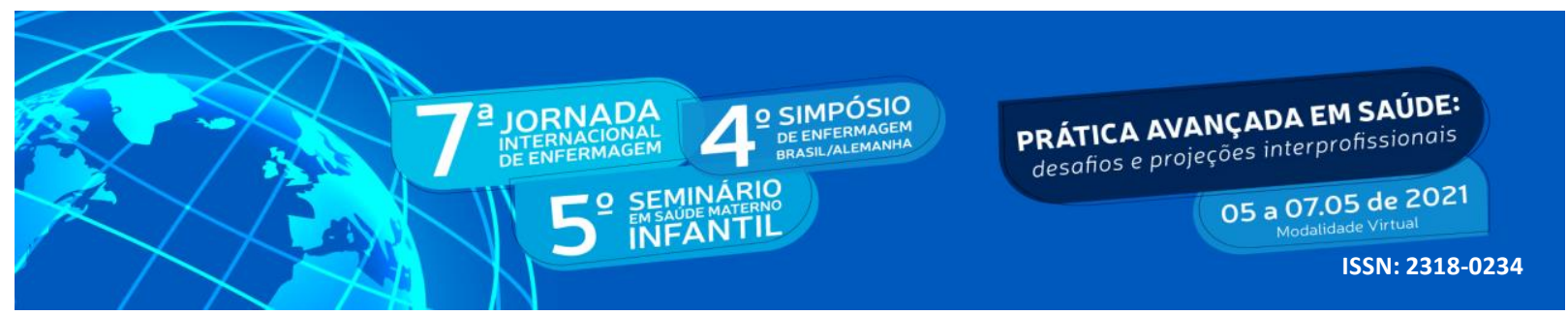

acerca da amamentação evidenciou que as gestantes compreenderam e praticaram os conhecimentos adquiridos após o parto (PIRO; AHMED, 2020). Ademais, destaca-se que as gestantes que participam dos grupos durante o pré-natal detêm mais conhecimento e segurança sobre o parto, amamentação e cuidados com recém-nascido, (LIVRAMENTO et al., 2019).

No mais, ressalta-se que este estudo apresentou como limitações o quantitativo de fontes consultadas, o tempo do recorte temporal, tal como a escassez de artigos que abordassem as inferências das ações do enfermeiro no cuidado à gestante na APS, restringindo a análise sobre esse aspecto. Contudo, este estudo se mostra relevante por reforçar a importância do cuidado do enfermeiro à gestante ao longo do pré-natal, fornecendo evidências para a continuidade da oferta de uma assistência de qualidade.

\section{CONCLUSÃO}

Conclui-se que as principais ações realizadas pelo enfermeiro, na atenção primária à saúde, são: acolhimento humanizado, prescrição e orientação para uso de medicamentos, orientações nutricionais e orientações sobre cuidados com o bebê, solicitação de exames, encaminhamento para atenção secundária e educação em saúde, e práticas de educação em saúde por meio de atividades educativas realizadas em grupo ou individualmente, tal como orientações referentes à nutrição, cuidados com o bebê, amamentação, repouso e posicionamento para dormir.

Destarte, ressalta-se a contribuição desse estudo para a enfermagem por contribuir para a compreensão das ações do enfermeiro, enquanto membro de uma equipe multiprofissional, acerca das estratégias utilizadas para promoção da saúde materno-infantil e prevenção de agravos a essa clientela.

\section{REFERÊNCIAS}

AGYEMAN-YEBOAH, J; KORSAH, K. A; OKRAH, J. Factors that influence the clinical utilization of the nursing process at a hospital in Accra, Ghana. BMC Nurs, v. 16, n. 1, p. 30, 2017. 


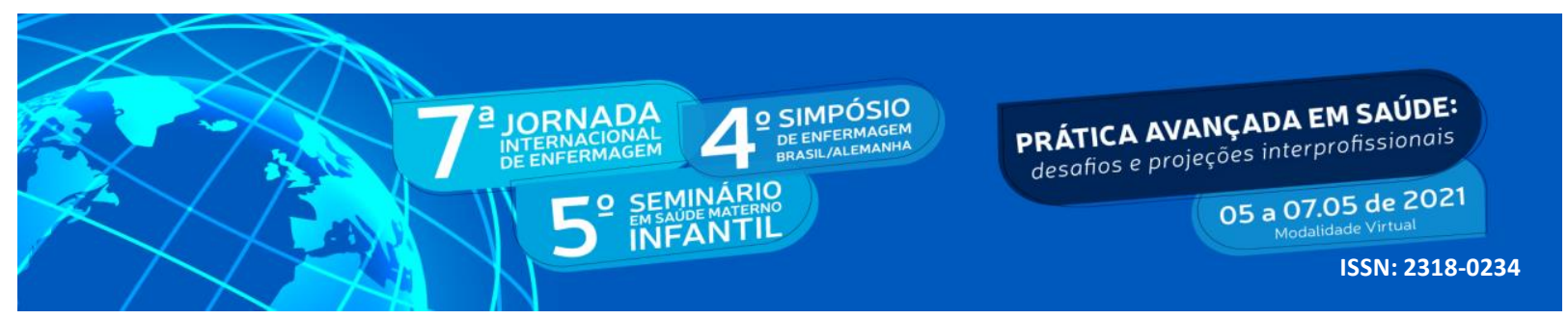

ARAÚJO, A. S. et al. The context of the pregnant woman in the situation of street and vulnerability: its look at the pre-natal. Rev enferm UFPE on line., v. 11, supl. 10, p. 410310, 2017.

BALSELLS, M. M. D. et al. Evaluation of prenatal care process for habitual-risk pregnant women. Acta paul. enferm., v. 31, n. 3, p. 247-254, 2018.

BORTOLI, C. F. C. et al. Factors that enable the performance of nurses in prenatal. J. res.: fundam. care. onlin., v. 9, n. 4, p. 978-983, 2017.

BRASIL. Ministério da Saúde. Pré-natal e puerpério: atenção qualificada e humanizada. Manual técnico. Brasília: Ministério da Saúde, 2006.

CARVALHO, L. A. et al. The use of technologies in nursing work: integrative review. J Nurs Health. v. 8, n. 1, e188104, 2018.

CHAVES, I. S. et al. Pre-natal consultation of nursing: satisfaction of pregnant women. $\mathbf{R}$. pesq.: cuid. fundam. online, v. 12, p. 814-819, 2020.

DIAS, E. G. et al. Nurses' actions in prenatal care and the importance attributed by pregnant women. Revista Sustinere, v. 6, n. 1, p. 52-62, 2018.

DYESS-NUGENT P. Nurses' unique opportunity to promote patient engagement in prenatal care. Nurs Forum. v. 53, n. 1, p. 61-68, 2018.

FERREIRA, S. R. S; PERICO, L. A. D; DIAS, V. R. F. G. The complexity of the work of nurses in Primary Health Care. Rev. Bras. Enferm., v. 71, supl. 1, p. 704-709, 2018.

IPUCHIMA, J. R; SOUZA, A. C; WEIS, A. H. Nurse's practice in the primary health care: an integrative review. J Nurs Health., v. 7, n. 3, e177303, 2017.

GARCIA, E. S. G. F. et al. The Nursing Care Actions Toward the Pregnant women: Challenging the Primary Health Care. J. res.: fundam. care. online v. 10, n. 3, p. 863-870, 2018.

GOMES, C. B. A. et al. Prenatal nursing consultation: narratives of pregnant women and nurses. Texto contexto - enferm., v. 28, e20170544, 2019.

KIM, S. Y; KIM, H. W. Prenatal nursing intervention studies published in Korean nursing journals: a scoping review. Korean Journal of Women Health Nursing, v. 26, n. 2, p. 109$119,2020$.

LIMA, K. M. S. G. et al. Nursing Care at High Risk Prenatal Care. Braz. J. Hea. Rev., v. 2, n. 4, p. 3183-3197, 2019. 


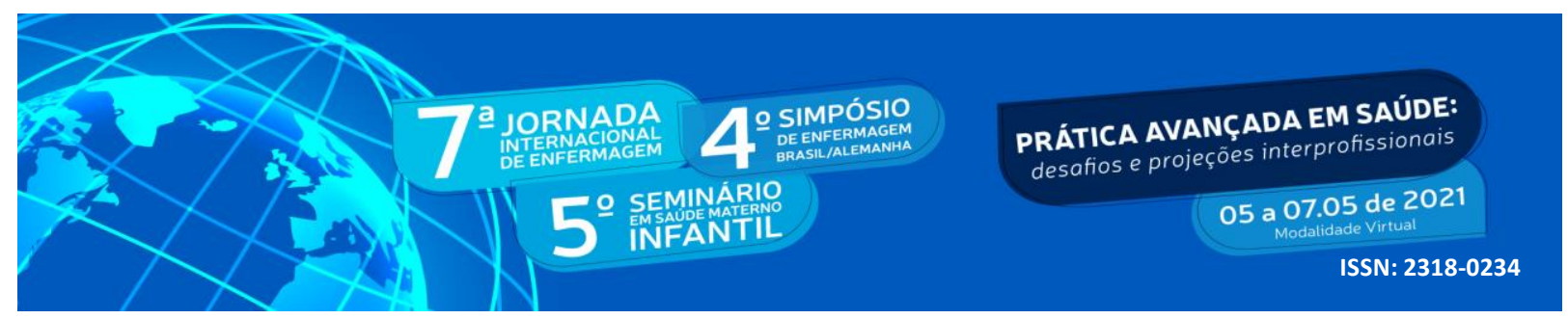

LIU, L. et al. Developing a prenatal nursing care International Classification for Nursing Practice catalogue. International Nursing Review, v. 64, n. 3, p. 371-378, 2017.

LIVRAMENTO, D. V. P. et al. Percepções de gestantes acerca do cuidado pré-natal na atenção primária à saúde. Rev. Gaúcha Enferm., v. 40, e20180211, 2019.

MARQUES, B. L. et al. Orientações às gestantes no pré-natal: a importância do cuidado compartilhado na atenção primária em saúde. Esc. Anna Nery, v. 25, n. 1, e20200098, 2021.

MAYOR, M.S.S. et al. Avaliação dos Indicadores da Assistência Pré-Natal em Unidade de Saúde da Família, em um Município da Amazônia Legal. Revista Cereus, v.10, n.1, p.91-100, 2018.

PETERS, A. A. et al. Pregnant women using psychoactive substances attended by nurses in Primary Health Care. SMAD, Rev Eletrônica Saúde Mental Álcool Drog, v. 16, n. 2, p. 6674, 2020.

PIRO, S. S; AHMED, H. M. Impacts of antenatal nursing interventions on mothers' breastfeeding self-efficacy: an experimental study. BMC Pregnancy and Childbirth, v. 20, n. 19, p. 1-12, 2020.

PIRO, S. S; AHMED, H. M. The Effectiveness of Antenatal Nursing Intervention on Initiation, Exclusivity, and Continuity of Breastfeeding. Sys Rev Pharm, v. 11, n. 1, p. 515$520,2020$.

ROCHA, C. G. G. et al. Social determinants of health in prenatal nursing consultation. Rev enferm UFPE on line., v. 13, e241571, 2019.

SIMÃO, A. M. S. et al. Management of prenatal nursing care at a Health Center in Angola. Rev Bras Enferm., v. 72, suppl 1, p. 136-43, 2019.

SOUSA, J. A. S. et al. Knowledge and perceptions on toxoplasmosis among pregnant women and nurses who provide prenatal in primary care. Rev. Inst. Med. trop. S. Paulo, v. 59, e31, 2017.

UNITED NATIONS ORGANIZATION (UNO). The Millennium Development Goals Report 2015. New York: United Nations, 2015.

WORLD HEALTH ORGANIZATION (WHO). Recommendations on antenatal care for a positive pregnancy experience. Geneva: World Health Organization, p. 172, 2016. 